\title{
Adequacy of nutritional intake during pregnancy in relation to prepregnancy BMI: results from the 3D Cohort Study
}

\author{
Lise Dubois $^{1 *}$, Maikol Diasparra ${ }^{1}$, Brigitte Bédard ${ }^{1}$, Cynthia K. Colapinto ${ }^{2,3}$, Bénédicte Fontaine-Bisson ${ }^{4,5}$, \\ Richard E. Tremblay ${ }^{6,7}$ and William D. Fraser ${ }^{2,3}$ \\ ${ }^{1}$ School of Epidemiology and Public Health, University of Ottawa, Ottawa, ON, Canada K1G $5 Z 3$ \\ ${ }^{2}$ Sainte-Justine University Hospital Research Centre, University of Montreal, Montreal, QC, Canada H3T 1C5 \\ ${ }^{3}$ Centre de recherche du Centre hospitalier universitaire de Sherbrooke, Sherbrooke, QC, Canada J1H $5 \mathrm{~N} 4$ \\ ${ }^{4}$ School of Nutrition Sciences, University of Ottawa, Ottawa, ON, Canada K1N 6N5 \\ ${ }^{5}$ Institut de recherche de l'Hôpital Montfort, Ottawa, ON, Canada K1K OT1 \\ ${ }^{6}$ Departments of Pediatrics and Psychology, University of Montreal, Montreal, QC, Canada H3T 1J7 \\ ${ }^{7}$ School of Public Heath, Physiotherapy and Sports Science, University College Dublin, Belfield, Dublin 4, Republic of Ireland \\ (Submitted 26 April 2017 - Final revision received 5 April 2018 - Accepted 19 April 2018)
}

\section{Abstract}

Our study compares adequacy of nutritional intakes among pregnant women with different prepregnancy BMI and explores associations between nutritional intakes during pregnancy and both prepregnancy BMI and gestational weight gain (GWG). We collected dietary information from a large cohort of pregnant Canadian women ( $n$ 861) using a 3-d food record. We estimated usual dietary intakes of energy (E), macronutrients and micronutrients using the National Cancer Institute method. We also performed Pearson's correlations between nutritional intakes and both prepregnancy BMI and GWG. In all BMI categories, intakes considered suboptimal (by comparison with estimated average requirements) were noted for $\mathrm{Fe}$, vitamin $\mathrm{D}$, folate, vitamin $\mathrm{B}_{6}, \mathrm{Mg}, \mathrm{Zn}$, Ca and vitamin $\mathrm{A}$. Total fat intakes were above the acceptable macronutrient distribution range (AMDR) for 36\% of the women. A higher proportion of obese women had carbohydrate intakes (as \%E) below the AMDR $(v$. normal-weight and overweight women; $19 v .9 \%)$ and $\mathrm{Na}$ intakes above the tolerable upper intake level ( $v$. other BMI categories; $90 v \cdot 77-78 \%$ ). In all BMI categories, median intakes of $\mathrm{K}$ and fibre were below adequate intake. Intakes of several nutrients (adjusted for energy) were correlated with BMI. Correlations were detected between energy-adjusted nutrient intakes and total GWG and were, for the most part, specific to certain BMI categories. Overweight and obese pregnant women appear to be the most nutritionally vulnerable. Nutrition interventions are needed to guide pregnant women toward their optimal GWG while also meeting their nutritional requirements.

\section{Key words: Nutrition: Diets: Pregnancy: Gestational weight gain: Prepregnancy BMI}

The worldwide obesity epidemic has seen the proportion of pregnant women with excess weight rise to unprecedented levels ${ }^{(1)}$. Moreover, because the prevalence of obesity has risen more rapidly than the prevalence of overweight, an increasing proportion of pregnant women are obese ${ }^{(1)}$. In 2012-2013, almost half of Canadian women of reproductive age (18-39 years) were overweight $(25 \%)$ or obese $(23 \%)^{(2)}$. This situation is alarming because maternal obesity is associated with adverse pregnancy and health outcomes for mothers and can have longterm health consequences for children ${ }^{(3,4)}$.

Another source of concern is excessive gestational weight gain (GWG). It affects large proportions of women in all prepregnancy BMI categories. Recent studies in the USA and Canada have estimated that up to half of normal-weight women, and up to two-thirds of overweight $\left(25 \mathrm{~kg} / \mathrm{m}^{2} \leq\right.$ $\left.\mathrm{BMI}<30 \mathrm{~kg} / \mathrm{m}^{2}\right)$ or obese $\left(\mathrm{BMI} \geq 30 \mathrm{~kg} / \mathrm{m}^{2}\right)$ women, have excessive $\mathrm{GWG}^{(5-7)}$. Excessive GWG correlates with adverse outcomes (e.g. fetal macrosomia), independently of prepregnancy $\mathrm{BMI}^{(6,8)}$. Obese women with excessive GWG are thus particularly at risk for pregnancy complications, and their children are at risk for short- and long-term health problems such as obesity, diabetes and other chronic diseases ${ }^{(4,9,10)}$. Inadequate GWG is also a public health concern because of its links to preterm birth, low birth weight and fetal growth restriction, three conditions that may also be related to obesity, metabolic dysfunction and other chronic diseases among offspring later in life $\mathrm{e}^{(10,11)}$. To respond to these issues, in 2009 , the US Institute of Medicine (IOM) revised its GWG recommendations ${ }^{(5)}$. The revised recommendations have been adopted by many countries, including Canada ${ }^{(12)}$. Overweight and obese women are advised to gain less weight, and underweight women $\left(\mathrm{BMI}<18.5 \mathrm{~kg} / \mathrm{m}^{2}\right)$ to gain more weight than normal-weight

Abbreviations: AMDR, acceptable macronutrient distribution range; GWG, gestational weight gain; IOM, Institute of Medicine; UL, tolerable upper intake level.

* Corresponding author: Professor L. Dubois, email ldubois@uottawa.ca 
women $\left(18.5 \mathrm{~kg} / \mathrm{m}^{2} \leq \mathrm{BMI}<25 \mathrm{~kg} / \mathrm{m}^{2}\right)$, during pregnancy. The IOM-recommended GWG ranges from 12.5 to $18.0 \mathrm{~kg}$ for underweight women, 11.5 to $16.0 \mathrm{~kg}$ for normal-weight women, 7.0 to $11.5 \mathrm{~kg}$ for overweight women and 5.0 to $9 \cdot 0 \mathrm{~kg}$ for obese women ${ }^{(5)}$.

Nutritional requirements, and, therefore, dietary reference intakes (DRI) for many nutrients, increase during pregnancy ${ }^{(4,13)}$. Adjusting maternal dietary intake to align with both recommended GWG levels and DRI can prove challenging, particularly for overweight or obese pregnant women who are encouraged to limit their GWG. As a consequence, enhancing the nutrient density of dietary intakes is of the utmost importance to ensure that maternal and fetal nutritional needs are met during this crucial period ${ }^{(4)}$. Recently, however, a meta-analysis has reported poor compliance with nutritional recommendations during pregnancy in industrialised countries ${ }^{(14,15)}$. A recent study we conducted estimated usual intakes of energy and of several macro- and micro-nutrients among a large cohort of pregnant women ${ }^{(16)}$. The results identified a number of nutrient inadequacies in dietary intakes ${ }^{(16)}$.

Several studies have reported that women who enter pregnancy with a higher BMI tend to have less healthy dietary patterns throughout pregnancy ${ }^{(17-21)}$. Obese women in particular have been found to consume fewer fibre sources (e.g. vegetables ${ }^{(19)}$, fruit $^{(22)}$, whole grains ${ }^{(22)}$ ), whereas women with a lower BMI were more likely to exhibit health-conscious dietary patterns during pregnancy $^{(18)}$. We may thus reasonably expect that adequacy of nutritional intakes and nutrient density also vary according to prepregnancy BMI. A fuller appreciation of these differences may prove particularly helpful in identifying priority areas for dietary interventions. Interventions could then be tailored to the needs of pregnant women in different BMI categories to whom different GWG recommendations would apply.

So far, studies investigating dietary intakes during pregnancy relative to prepregnancy BMI have mainly used composite measures of diet, which has allowed identifying differential dietary patterns by means of factor analysis, cluster analysis and index analysis $^{(17-21)}$. Few studies have focused on nutritional intakes during pregnancy relative to prepregnancy BMI. In a small study conducted in the UK in 2002-2003 ( $n$ 72), Derbyshire et al. ${ }^{(23)}$ collected dietary information using a 4-to-7-d weighed-food diary and performed analyses of correlations between prepregnancy BMI and nutritional intakes (in absolute values) in the first trimester of pregnancy. The authors did not, however, consider energy-adjusted nutrient intakes ${ }^{(23)}$. Moreover, since the revised IOM-GWG recommendations were published, to our knowledge no large-scale study has documented nutritional intakes (including a broad range of nutrients) among pregnant women with different prepregnancy BMI relative to DRI compliance.

A primary objective of our study is, therefore, to compare nutritional intake adequacy among women across prepregnancy BMI categories and to assess associations between nutritional intakes (energy adjusted) during pregnancy and prepregnancy BMI. As diet is a modifiable risk factor for GWG and may to a certain extent determine compliance with GWG recommendations, we will also explore potential associations between nutritional intakes during pregnancy and GWG for different prepregnancy BMI categories.

\section{Methods}

\section{Studied population}

The present study was performed using data from a pregnancy/ birth cohort, the 3D Cohort Study (Design, Develop, Discover). This cohort study has been described in detail elsewhere ${ }^{(24)}$. In brief, a total of 2366 pregnant women were recruited between May 2010 and August 2012 from nine obstetric clinics in urban areas in the Province of Quebec, Canada. The women taking part in the 3D Study were recruited in the first trimester of pregnancy ( 8 to $<14$ weeks) with follow-up visits in the second (20 to $<24$ weeks) and third (32 to $<34$ weeks) trimesters, and at delivery. The children were followed from birth to 2 years of age. All instances of data collection covered a broad range of dimensions (e.g. sociodemographic, environmental, behavioural, medical) and employed multiple instruments (e.g. face-to-face interviews, self-reported questionnaires, medical charts). This study was conducted according to the guidelines laid down in the Declaration of Helsinki. As such, all procedures involving human subjects were approved by the research ethics committee at the coordinating centre at Sainte-Justine's Hospital in Montreal, as well as academic and hospital ethics committees at all participating study sites. Written informed consent was obtained from all 3D Cohort Study participants upon recruitment.

Prepregnancy BMI (weight $(\mathrm{kg}) /$ height $\left(\mathrm{m}^{2}\right)$ ) was calculated from measured height and self-reported prepregnancy body weight captured during the first visit. Four categories of prepregnancy weight status were defined based on internationally accepted cut-offs for BMI: underweight $\left(<18.5 \mathrm{~kg} / \mathrm{m}^{2}\right)$, normal weight $\left(18.5-24.9 \mathrm{~kg} / \mathrm{m}^{2}\right)$, overweight $\left(25.0-29.9 \mathrm{~kg} / \mathrm{m}^{2}\right)$ and obese $\left(\geq 30 \cdot 0 \mathrm{~kg} / \mathrm{m}^{2}\right)$. GWG was calculated as the difference between weight measured in the 3 weeks before delivery and self-reported prepregnancy weight. GWG conformity with the IOM-recommended levels published in $2009^{(5)}$ was determined using the specific cut-offs for the four BMI categories and defined as comprising three levels (below, within, above).

Dietary intake was assessed from data gathered using a 3-d food record provided to participants on their second visit (i.e. in the second trimester). Participants were asked to record all food and drinks consumed on 2 weekdays and 1 weekend day during a week-long reference period. The use of food records allowed detailed information to be collected on both food products ingested and food preparation methods. Food quantities were estimated using common household items (e.g. measuring cups, tablespoons). Dietary information obtained from food records was coded by nutrition professionals trained in the use of Food Processor software, version 10.13.1 (ESHA Research, Inc.), which incorporated the latest version of the Canadian food composition database (Canadian Nutrient File $)^{(25)}$. Coding activities also included quality control procedures where accuracy was validated by reviewing $20 \%$ of the coded records and verifying extreme values. The coded information was then used to derive the energy and nutrient content of participants' diets. We also assessed the extent of misreporting by comparing the ratio of estimated energy intake (eEI):estimated BMR (eBMR) for pregnant women with plausible cut-off values derived from Goldberg's equations ${ }^{(26)}$. We assumed a physical activity level of 1.55 , and used CV for BMR, 
physical activity and within-subject energy intakes of 8.5, 15 and 23 , respectively. Participants having a eEI:eBMR ratio between 1.00 and 2.40 were identified as 'acceptable reporters' (which was the case for $93 \%$ of the pregnant women who provided complete and valid nutrition data from 3-d food records).

Only full-term pregnancies (37 weeks or more) were considered for inclusion in the study. To be eligible for the study, women also had to have provided information on prepregnancy weight, to have had their height measured (required for calculating BMI) and to have had final weight measured in the 3 weeks before delivery (to assess GWG). Out of 1319 eligible participants, 924 women reported $3 \mathrm{~d}$ of dietary intake on valid food records. Because the prevalence of energy misreporting is known to vary according to weight status, we used data from acceptable reporters only. Thus, nutritional analysis was conducted on a sample of 861 pregnant women.

\section{Statistical analyses}

We estimated usual dietary intakes of energy (E; kJ (kcal)), macronutrients and several other nutrients essential for a healthy pregnancy by using the National Cancer Institute method $^{(27)}$. For each nutrient, a non-linear mixed model was derived from the whole sample. We then computed the distribution of usual intakes for all women and separately for each prepregnancy BMI category. We applied a Box-Cox transformation to all nutritional variables before analysis as they were not normally distributed. Certain covariates (i.e. mother's age; ordinal food diary day (1st, 2nd or 3rd); weekday or weekend day; prepregnancy BMI category; and category of GWG relative to recommended levels (below, within or above)) were included in the models, when significant. Estimated distributions of usual intakes were used to calculate the proportions below estimated average requirement (EAR), above tolerable upper intake level (UL) for $\mathrm{Na}$ and below and above acceptable macronutrient distribution range (AMDR) for macronutrients

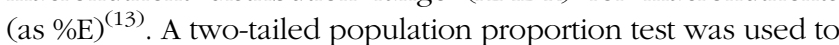
compare proportions across prepregnancy BMI categories. We also performed Pearson's correlations between energy and nutrient intakes (energy adjusted) and prepregnancy BMI. Finally, we computed Pearson's correlations between nutritional intakes (total and energy adjusted) and GWG for all participants and by prepregnancy BMI category. All analyses were conducted using SAS (version 9.4; SAS Institute); the significance level was set at 0.05 .

\section{Results}

Table 1 presents characteristics of the studied population. A majority of pregnant women had university degrees (63\%) and lived in middle-to-upper-income households (59\% reporting annual family incomes $\geq \$ 80000$ (Canadian dollars)). A quarter ( $27 \%$ ) of the women reported excess weight before pregnancy (BMI $\geq 25 \mathrm{~kg} / \mathrm{m}^{2}$ ). Relative to their prepregnancy BMI category, $17 \%$ of the women had a GWG below, whereas almost half (48\%) of the women had a GWG above, as per IOM
Table 1. Description of the studied population ( $n$ 861) (Numbers and percentages)

\begin{tabular}{|c|c|c|}
\hline Characteristics & $n$ & $\%$ \\
\hline \multicolumn{3}{|l|}{ Parity } \\
\hline 0 & 507 & 58.9 \\
\hline 1 & 263 & 30.5 \\
\hline 2 or more & 91 & $10 \cdot 6$ \\
\hline \multicolumn{3}{|l|}{ Smoking ( $n$ 860) } \\
\hline Yes & 99 & 11.5 \\
\hline No & 761 & 88.5 \\
\hline \multicolumn{3}{|c|}{ Gestational diabetes ( $n$ 860) } \\
\hline Yes & 69 & $8 \cdot 0$ \\
\hline No & 791 & $92 \cdot 0$ \\
\hline \multicolumn{3}{|l|}{ Excessive vomiting* } \\
\hline Yes & 76 & $8 \cdot 8$ \\
\hline No & 785 & $91 \cdot 2$ \\
\hline \multicolumn{3}{|l|}{ Age (years) } \\
\hline $19-24$ & 54 & $6 \cdot 3$ \\
\hline $25-29$ & 289 & 33.6 \\
\hline $30-34$ & 335 & 38.9 \\
\hline 35 or older & 183 & $21 \cdot 2$ \\
\hline \multicolumn{3}{|l|}{ Education ( $n$ 858) } \\
\hline High school or less & 47 & $5 \cdot 5$ \\
\hline College & 215 & $25 \cdot 1$ \\
\hline University & 358 & 41.7 \\
\hline Graduate school & 238 & $27 \cdot 7$ \\
\hline \multicolumn{3}{|c|}{ Annual family income ( $n$ 832) } \\
\hline$<\$ 30000$ & 61 & $7 \cdot 3$ \\
\hline$\$ 30000-59999$ & 133 & $16 \cdot 0$ \\
\hline$\$ 60000-79999$ & 147 & $17 \cdot 7$ \\
\hline$\$ 80000-99999$ & 198 & $23 \cdot 8$ \\
\hline$\geq \$ 100000$ & 293 & $35 \cdot 2$ \\
\hline \multicolumn{3}{|l|}{ Born in Canada ( $n$ 860) } \\
\hline Yes & 630 & $73 \cdot 3$ \\
\hline No & 230 & $26 \cdot 7$ \\
\hline \multicolumn{3}{|c|}{ Prepregnancy BMI category† } \\
\hline Underweight & 51 & $5 \cdot 9$ \\
\hline Normal weight & 575 & $66 \cdot 8$ \\
\hline Overweight & 147 & $17 \cdot 1$ \\
\hline Obese & 88 & $10 \cdot 2$ \\
\hline \multicolumn{3}{|c|}{ GWG relative to recommendations $\ddagger$} \\
\hline Below & 143 & $16 \cdot 6$ \\
\hline Within & 305 & $35 \cdot 4$ \\
\hline Above & 413 & $48 \cdot 0$ \\
\hline
\end{tabular}

GWG, gestational weight gain.

* Excessive vomiting with weight loss reported in either first or second trimester of pregnancy.

† Underweight: $\mathrm{BMI}<18.5 \mathrm{~kg} / \mathrm{m}^{2}$; normal weight: $18.5 \mathrm{~kg} / \mathrm{m}^{2} \leq \mathrm{BMl}<25.0 \mathrm{~kg} / \mathrm{m}^{2}$; overweight: $25.0 \mathrm{~kg} / \mathrm{m}^{2} \leq \mathrm{BMl}<30.0 \mathrm{~kg} / \mathrm{m}^{2}$; obese: $\mathrm{BMI} \geq 30.0 \mathrm{~kg} / \mathrm{m}^{2}$

‡ The Institute of Medicine (IOM)-recommended $\mathrm{GWG}^{(5)}$ ranges from 12.5 to $18.0 \mathrm{~kg}$ for underweight women, 11.5 to $16.0 \mathrm{~kg}$ for normal-weight women, 7.0 to $11.5 \mathrm{~kg}$ for overweight women and 5.0 to $9.0 \mathrm{~kg}$ for obese women.

recommendations. Prepregnancy BMI was also associated with GWG (see online Supplementary Table S1). A higher proportion of overweight and obese women ( $74 \%$ in both cases) had GWG values above levels recommended for their BMI categories than did normal-weight and underweight women (39 and $27 \%$ respectively). At the other end of the spectrum, one in five normal-weight and underweight women $(20 \%$ in both cases) and about one in ten obese women (11\%) had GWG values below recommended levels for their BMI categories. Overweight women had a low prevalence of inadequate GWG.

Table 2 presents estimated usual dietary intakes of energy and various nutrients (medians and interquartile ranges) during pregnancy relative to prepregnancy BMI categories, whereas Table 3 shows comparisons with DRI values. EAR comparisons 
Table 2. Usual dietary intakes of energy and nutrients during pregnancy* for all participants $(n 861) \dagger$ and by prepregnancy BMI category $\ddagger$ (Medians and interquartile ranges (IQR))

\begin{tabular}{|c|c|c|c|c|c|c|c|c|c|c|}
\hline & \multicolumn{2}{|r|}{ All } & \multicolumn{2}{|c|}{ Underweight ( $n 51)$} & \multicolumn{2}{|c|}{ Normal weight ( $n$ 575) } & \multicolumn{2}{|c|}{ Overweight ( $n$ 147) } & \multicolumn{2}{|c|}{ Obese ( $n 88)$} \\
\hline & Median & IQR & Median & IQR & Median & IQR & Median & IQR & Median & IQR \\
\hline Energy (kJ) & $9313 \cdot 2$ & $8251 \cdot 7 ; 10448.7$ & 9304.8 & $8209 \cdot 4 ; 34348 \cdot 2$ & $9315 \cdot 2$ & $8249 \cdot 6 ; 10448.7$ & 9329.5 & $8276 ; 10446.6$ & 9265.9 & $8253 \cdot 3 ; 10421 \cdot 1$ \\
\hline Energy (kcal) & 2225.9 & $1972 \cdot 2 ; 2497 \cdot 3$ & 2223.9 & $1962 \cdot 1 ; 2510 \cdot 4$ & $2226 \cdot 5$ & $1971.7 ; 2497.3$ & 2229.8 & $1978.0 ; 2496.8$ & $2214 \cdot 6$ & $1972 \cdot 6 ; 2490 \cdot 7$ \\
\hline Protein (\%E) & $16 \cdot 5$ & $14.9 ; 18.2$ & $16 \cdot 4$ & $14.8 ; 18.2$ & $16 \cdot 4$ & $14.9 ; 18.1$ & $16 \cdot 5$ & $14.9 ; 18 \cdot 2$ & $17 \cdot 3$ & $15 \cdot 7 ; 19 \cdot 1$ \\
\hline Carbohydrate (\%E) & 52.1 & $48.4 ; 55.8$ & $52 \cdot 3$ & $48 \cdot 5 ; 56 \cdot 2$ & 52.4 & $48 \cdot 7 ; 56.0$ & 52.4 & $48.8 ; 56.0$ & 49.6 & $46 \cdot 1 ; 53.3$ \\
\hline Total sugar (\%E) & 21.3 & $18 \cdot 4 ; 24 \cdot 3$ & 21.3 & $18 \cdot 3 ; 24 \cdot 5$ & 21.3 & $18.4 ; 24.3$ & 21.3 & $18 \cdot 5 ; 24 \cdot 3$ & $21 \cdot 2$ & $18 \cdot 4 ; 24.2$ \\
\hline Total fat (\%E) & 33.3 & $30.1 ; 36.5$ & 33.3 & $29 \cdot 9 ; 36.7$ & 33.3 & $30.0 ; 36.5$ & 33.4 & $30.1 ; 36.5$ & 33.2 & $30.1 ; 36.5$ \\
\hline SFA (\%E) & 11.8 & $10 \cdot 2 ; 13.5$ & 11.8 & $10 \cdot 1 ; 13 \cdot 6$ & 11.8 & $10 \cdot 2 ; 13.5$ & 11.8 & $10 \cdot 2 ; 13.5$ & 11.7 & $10 \cdot 2 ; 13.5$ \\
\hline MUFA (\%E) & $12 \cdot 2$ & $10 \cdot 5 ; 14.0$ & $12 \cdot 2$ & $10 \cdot 5 ; 14 \cdot 1$ & $12 \cdot 2$ & $10.5 ; 14.0$ & $12 \cdot 2$ & $10 \cdot 6 ; 14.0$ & $12 \cdot 1$ & $10.5 ; 13.9$ \\
\hline PUFA (\%E) & $5 \cdot 8$ & $4.9 ; 6.9$ & $5 \cdot 8$ & $4.9 ; 6.9$ & $5 \cdot 8$ & $4.9 ; 6.9$ & $5 \cdot 8$ & $4.9 ; 6.9$ & $5 \cdot 8$ & $4.9 ; 6.8$ \\
\hline Protein $(\mathrm{g})$ & 91.4 & $79.8 ; 103.7$ & $90 \cdot 3$ & $78.6 ; 103.3$ & 90.6 & $79 \cdot 1 ; 102 \cdot 7$ & 90.7 & $79 \cdot 4 ; 102 \cdot 7$ & 98.6 & $86.9 ; 111.3$ \\
\hline Carbohydrate (g) & $287 \cdot 7$ & $250.4 ; 328.0$ & 287.5 & $249 \cdot 0 ; 330 \cdot 1$ & $288 \cdot 0$ & $250.5 ; 328.1$ & $287 \cdot 6$ & $250.9 ; 327.3$ & 285.8 & $250 \cdot 1 ; 327 \cdot 6$ \\
\hline Dietary fibre $(\mathrm{g})$ & 23.5 & $19.3 ; 28.5$ & 24.0 & $19 \cdot 7 ; 29.2$ & $24 \cdot 1$ & $19 \cdot .7 ; 29 \cdot 1$ & $22 \cdot 1$ & $18 \cdot 1 ; 26 \cdot 7$ & $22 \cdot 1$ & $18 \cdot 3 ; 26 \cdot 8$ \\
\hline Total fat $(\mathrm{g})$ & 82.7 & $69 \cdot 3 ; 97.2$ & 80.0 & $66.5 ; 95.0$ & 81.8 & $68 \cdot 6 ; 96 \cdot 1$ & 84.6 & $71.2 ; 98.9$ & $87 \cdot 1$ & $74 \cdot 1 ; 102 \cdot 4$ \\
\hline n-6 Linoleic acid (g) & $12 \cdot 4$ & $9.8 ; 15.5$ & 11.9 & $9 \cdot 3 ; 15 \cdot 1$ & $12 \cdot 3$ & $9 \cdot 7 ; 15 \cdot 3$ & $12 \cdot 7$ & $10 \cdot 1 ; 15 \cdot 8$ & $13 \cdot 3$ & $10 \cdot 6 ; 16 \cdot 7$ \\
\hline$n-3$ Linolenic acid (g) & 1.5 & $1.1 ; 1.9$ & 1.5 & $1 \cdot 1 ; 2.0$ & 1.5 & $1.1 ; 1.9$ & 1.5 & $1.1 ; 1.9$ & 1.5 & $1.1 ; 1.9$ \\
\hline Cholesterol (mg) & 278.9 & $221 \cdot 8 ; 344.9$ & $265 \cdot 1$ & $209.4 ; 332.3$ & 273.9 & $218 \cdot 1 ; 338.6$ & 288.9 & $230.6 ; 353.7$ & 303.1 & $244.9 ; 374.4$ \\
\hline Vitamin A (RAE, $\mu \mathrm{g})$ & $882 \cdot 2$ & $691.9 ; 1113.1$ & $880 \cdot 6$ & $684.9 ; 1124.9$ & $882 \cdot 7$ & $691.5 ; 1113.0$ & $885 \cdot 3$ & $696 \cdot 0 ; 1112.5$ & $873 \cdot 2$ & $692 \cdot 2 ; 1107 \cdot 1$ \\
\hline Thiamine (mg) & 1.9 & $1.6 ; 2.3$ & 1.9 & $1 \cdot 6 ; 2.3$ & 1.9 & $1 \cdot 6 ; 2 \cdot 3$ & 1.9 & $1.6 ; 2.3$ & 1.9 & $1 \cdot 6 ; 2 \cdot 3$ \\
\hline Riboflavin (mg) & $2 \cdot 3$ & $2 \cdot 0 ; 2.7$ & $2 \cdot 3$ & $2 \cdot 0 ; 2 \cdot 7$ & $2 \cdot 3$ & $2 \cdot 0 ; 2 \cdot 7$ & $2 \cdot 3$ & $2 \cdot 0 ; 2 \cdot 7$ & $2 \cdot 3$ & $2 \cdot 0 ; 2 \cdot 7$ \\
\hline Niacin (NE) & 38.4 & $34.4 ; 42.5$ & 38.0 & $34.0 ; 42 \cdot 3$ & 38.0 & $34 \cdot 2 ; 42 \cdot 1$ & 38.1 & $34 \cdot 3 ; 42 \cdot 1$ & 41.1 & $37 \cdot 2 ; 45 \cdot 4$ \\
\hline Vitamin $B_{6}(m g)$ & 1.9 & $1 \cdot 6 ; 2 \cdot 2$ & 1.9 & $1 \cdot 6 ; 2 \cdot 2$ & 1.9 & $1.6 ; 2.2$ & 1.9 & $1.6 ; 2.2$ & 1.9 & $1 \cdot 6 ; 2.2$ \\
\hline Folate (DFE, $\mu \mathrm{g}$ ) & $466 \cdot 0$ & $388.6 ; 550.9$ & $465 \cdot 4$ & $385.6 ; 555.0$ & $466 \cdot 2$ & $388.4 ; 550.9$ & $467 \cdot 2$ & $390 \cdot 3 ; 550 \cdot 7$ & 462.5 & $388 \cdot 7 ; 548 \cdot 8$ \\
\hline Vitamin $B_{12}(\mu \mathrm{g})$ & $5 \cdot 0$ & $3.9 ; 6 \cdot 2$ & 5.0 & $3.9 ; 6 \cdot 3$ & 5.0 & $3.9 ; 6.2$ & $5 \cdot 0$ & $4.0 ; 6 \cdot 2$ & 4.9 & $3.9 ; 6.2$ \\
\hline Vitamin C (mg) & $162 \cdot 7$ & $114 \cdot 3 ; 220 \cdot 3$ & $162 \cdot 3$ & $112 \cdot 5 ; 223 \cdot 2$ & $162 \cdot 8$ & $114 \cdot 2 ; 220 \cdot 3$ & 163.5 & $115 \cdot 3 ; 220 \cdot 1$ & $160 \cdot 4$ & $114.4 ; 218 \cdot 8$ \\
\hline Vitamin D $(\mu \mathrm{g})$ & 5.6 & $4.0 ; 7.4$ & 5.5 & $3.9 ; 7.5$ & $5 \cdot 6$ & $4.0 ; 7.4$ & $5 \cdot 6$ & $4.0 ; 7.4$ & 5.5 & $4.0 ; 7.4$ \\
\hline $\mathrm{Ca}(\mathrm{mg})$ & $1210 \cdot 4$ & $976 \cdot 0 ; 1472 \cdot 0$ & 1208.5 & $966.9 ; 1484.8$ & 1211.0 & $975.5 ; 1472.0$ & 1214.0 & $981.2 ; 1471.5$ & 1199.7 & $976 \cdot 3 ; 1465 \cdot 6$ \\
\hline $\mathrm{Fe}(\mathrm{mg})$ & $15 \cdot 3$ & $13 \cdot 1 ; 17 \cdot 7$ & $15 \cdot 3$ & $13 \cdot 0 ; 17 \cdot 9$ & $15 \cdot 3$ & $13 \cdot 1 ; 17 \cdot 7$ & $15 \cdot 3$ & $13 \cdot 2 ; 17 \cdot 7$ & $15 \cdot 2$ & $13 \cdot 1 ; 17 \cdot 7$ \\
\hline $\mathrm{Mg}(\mathrm{mg})$ & 370.3 & $314.6 ; 433.2$ & 369.5 & $311.9 ; 435.0$ & $370 \cdot 2$ & $314.4 ; 433.3$ & 371.7 & $316 \cdot 1 ; 433.8$ & 368.4 & $314.2 ; 430.4$ \\
\hline$P(\mathrm{mg})$ & 1541.3 & $1320.4 ; 1784.3$ & $1539 \cdot 6$ & $1311 \cdot 7 ; 1796 \cdot 2$ & 1541.9 & $1319 \cdot 9 ; 1784 \cdot 3$ & $1544 \cdot 7$ & $1325 \cdot 4 ; 1783.8$ & 1531.3 & $1320 \cdot 7 ; 1778 \cdot 3$ \\
\hline $\mathrm{Na}(\mathrm{mg})$ & 2877.5 & $2383 \cdot 1 ; 3419 \cdot 3$ & 2819.9 & $2332.1 ; 3389.5$ & 2841.3 & $2354.7 ; 3371.1$ & $2827 \cdot 1$ & $2353 \cdot 2 ; 3352 \cdot 1$ & 3243.5 & $2730 \cdot 7 ; 3831 \cdot 4$ \\
\hline $\mathrm{K}(\mathrm{mg})$ & $3409 \cdot 3$ & $2935.2 ; 3920 \cdot 4$ & 3405.5 & $2916.4 ; 3945.0$ & 3410.5 & $2934.2 ; 3920.3$ & 3416.5 & $2945.9 ; 3919.3$ & 3388.1 & $2935.8 ; 3907.9$ \\
\hline $\mathrm{Zn}(\mathrm{mg})$ & 11.9 & $10 \cdot 2 ; 13.8$ & $10 \cdot 8$ & $9.2 ; 12.7$ & 11.8 & $10.1 ; 13.7$ & 11.8 & $10 \cdot 2 ; 13.7$ & 12.9 & $11.1 ; 15.0$ \\
\hline
\end{tabular}

E, energy; RAE, retinol activity equivalent; NE, niacin equivalent; DFE, dietary folate equivalent.

* Usual intakes were estimated by using the National Cancer Institute method ${ }^{(27)}$. For each nutrient, a non-linear mixed model was derived for the whole sample. The distribution of usual intakes was then computed for all participants and separately for each prepregnancy BMI category. A Box-Cox transformation was applied to all nutritional variables before analysis. Certain covariates (e.g. mother's age; 1st, 2nd or 3rd day of the food diary; weekday/weekend day) were included in the models when relevant.

† Acceptable reporters only (ratio of energy intake:BMR between 1.00 and 2.40 ).

‡ Underweight: BMI $<18.5 \mathrm{~kg} / \mathrm{m}^{2}$; normal weight: $18.5 \mathrm{~kg} / \mathrm{m}^{2} \leq \mathrm{BMI}<25.0 \mathrm{~kg} / \mathrm{m}^{2}$; overweight: $25.0 \mathrm{~kg} / \mathrm{m}^{2} \leq \mathrm{BMl}<30.0 \mathrm{~kg} / \mathrm{m}^{2} ;$ obese: $\mathrm{BMl} \geq 30.0 \mathrm{~kg} / \mathrm{m}^{2}$. 
Table 3. Adequacy of nutritional intakes during pregnancy† relative to dietary reference intakes (DRI) for all participants $(n$ 861) $\ddagger$ and by prepregnancy BMl category§

(Percentages below or above selected DRI values for pregnancy)

\begin{tabular}{|c|c|c|c|c|c|c|c|c|c|c|c|}
\hline & \multirow[b]{2}{*}{$\begin{array}{l}\text { DRI values } \\
\text { (19 years }+ \text { ) }\end{array}$} & \multicolumn{2}{|c|}{ All } & \multicolumn{2}{|c|}{ Underweight ( $n$ 51) } & \multicolumn{2}{|c|}{ Normal weight ( $n$ 575) } & \multicolumn{2}{|c|}{ Overweight ( $n$ 147) } & \multicolumn{2}{|c|}{ Obese $(n 88)$} \\
\hline & & $\begin{array}{l}\text { AMDR or } \\
\text { EAR }(\%<)\end{array}$ & $\begin{array}{l}\text { AMDR or } \\
\text { UL }(\%>)\end{array}$ & $\begin{array}{l}\text { AMDR or } \\
\text { EAR }(\%<)\end{array}$ & $\begin{array}{l}\text { AMDR or } \\
\text { UL }(\%>)\end{array}$ & $\begin{array}{l}\text { AMDR or } \\
\text { EAR }(\%<)\end{array}$ & $\begin{array}{l}\text { AMDR or } \\
\text { UL }(\%>)\end{array}$ & $\begin{array}{l}\text { AMDR or } \\
\text { EAR }(\%<)\end{array}$ & $\begin{array}{l}\text { AMDR or } \\
\text { UL }(\%>)\end{array}$ & $\begin{array}{l}\text { AMDR or } \\
\text { EAR }(\%<)\end{array}$ & $\begin{array}{l}\text { AMDR or } \\
\text { UL }(\%>)\end{array}$ \\
\hline Protein (\%E) & AMDR $10-35$ & 0.1 & 0.0 & 0.1 & 0.0 & 0.1 & 0.0 & 0.1 & 0.0 & 0.0 & 0.0 \\
\hline Total fat (\%E) & AMDR $20-35$ & 0.3 & 36.2 & 0.4 & $36 \cdot 2$ & 0.3 & $36 \cdot 2$ & 0.3 & $36 \cdot 3$ & 0.3 & 35.6 \\
\hline Carbohydrate (\%E) & AMDR $45-65$ & 9.6 & 1.0 & 8.5 & 1.0 & $8 \cdot 6^{*}$ & $1 \cdot 1$ & $8 \cdot 5^{*}$ & $1 \cdot 2$ & $18 \cdot 8$ & 0.3 \\
\hline Carbohydrate (g) & EAR 135 & 0.0 & & 0.1 & & 0.0 & & 0.0 & & 0.0 & \\
\hline Vitamin A (RAE, $\mu \mathrm{g})$ & EAR 550 & 9.8 & & 9.6 & & 9.8 & & $9 \cdot 7$ & & 9.8 & \\
\hline Thiamine (mg) & EAR 1.2 & $4 \cdot 2$ & & 4.5 & & $4 \cdot 2$ & & $4 \cdot 1$ & & $4 \cdot 3$ & \\
\hline Riboflavin (mg) & EAR 1.2 & 0.4 & & 0.5 & & 0.3 & & 0.4 & & 0.3 & \\
\hline Niacin (NE) & EAR 14 & 0.0 & & 0.0 & & 0.0 & & 0.0 & & 0.0 & \\
\hline Vitamin $B_{6}(\mathrm{mg})$ & EAR 1.6 & 24.5 & & $25 \cdot 4$ & & 24.6 & & $24 \cdot 1$ & & 24.5 & \\
\hline Folate (DFE, $\mu \mathrm{g}$ ) & EAR 520 & $66 \cdot 8$ & & 66.4 & & 66.8 & & $66 \cdot 6$ & & 67.5 & \\
\hline Vitamin $B_{12}(\mu \mathrm{g})$ & EAR $2 \cdot 2$ & $1 \cdot 1$ & & 1.2 & & 1.0 & & 1.2 & & $1 \cdot 1$ & \\
\hline Vitamin C (mg) & EAR 70 & $7 \cdot 2$ & & 7.3 & & $7 \cdot 2$ & & $7 \cdot 1$ & & $7 \cdot 2$ & \\
\hline Vitamin $D(\mu \mathrm{g})$ & EAR 10 & $92 \cdot 7$ & & 92.5 & & $92 \cdot 7$ & & 93.1 & & $92 \cdot 7$ & \\
\hline $\mathrm{Ca}(\mathrm{mg})$ & EAR 800 & $10 \cdot 5$ & & 10.5 & & $10 \cdot 6$ & & $10 \cdot 4$ & & $10 \cdot 5$ & \\
\hline $\mathrm{Fe}(\mathrm{mg})$ & EAR 22 & $95 \cdot 2$ & & 94.8 & & $95 \cdot 2$ & & 95.5 & & $95 \cdot 3$ & \\
\hline $\mathrm{Mg}(\mathrm{mg})$ & EAR 290॥ & $15 \cdot 8$ & & $16 \cdot 0$ & & $15 \cdot 9$ & & 15.5 & & $15 \cdot 6$ & \\
\hline$P(\mathrm{mg})$ & EAR 580 & 0.0 & & 0.0 & & 0.0 & & 0.0 & & 0.0 & \\
\hline $\mathrm{Na}(\mathrm{mg})$ & UL 2300 & & $78 \cdot 8$ & & $76 \cdot 6^{*}$ & & $77 \cdot 7^{*}$ & & $77 \cdot 4^{*}$ & & 90.2 \\
\hline $\mathrm{Zn}(\mathrm{mg})$ & EAR 9.5 & $16 \cdot 9$ & & 29.4 & & $17 \cdot 1$ & & $16 \cdot 8$ & & 8.5 & \\
\hline
\end{tabular}

AMDR, acceptable macronutrient distribution range; EAR, estimated average requirement; UL, tolerable upper intake level; E, energy; RAE, retinol activity equivalent; NE, niacin equivalent; DFE, dietary folate equivalent.

* Value significantly different from that for obese women $(P<0.05)$.

† Usual intakes were estimated by using the National Cancer Institute method ${ }^{(27)}$. For each nutrient, a non-linear mixed model was derived for the whole sample. The distribution of usual intakes was then computed for all participants and separately for each prepregnancy BMI category. A Box-Cox transformation was applied to all nutritional variables before analysis. Certain covariates (e.g. mother's age; 1st, 2nd or 3rd day of the food diary; weekday/weekend day) were included in the models when relevant. Estimated distributions of usual intakes served to calculate proportions below EAR, above UL (for Na) and below and above AMDR (for major macronutrients as \%E). A two-tailed population proportion test was used to compare proportions across prepregnancy BMI categories.

$\ddagger$ Acceptable reporters only (ratio of energy intake:BMR between 1.00 and 2.40 ).

$\S$ Underweight: $\mathrm{BMI}<18.5 \mathrm{~kg} / \mathrm{m}^{2}$; normal weight: $18.5 \mathrm{~kg} / \mathrm{m}^{2} \leq \mathrm{BMl}<25.0 \mathrm{~kg} / \mathrm{m}^{2}$; overweight: $25.0 \mathrm{~kg} / \mathrm{m}^{2} \leq \mathrm{BMl}<30.0 \mathrm{~kg} / \mathrm{m}^{2} ;$ obese: $\mathrm{BMI} \geq 30.0 \mathrm{~kg} / \mathrm{m}^{2}$.

II EAR value for 19-30-year-old pregnant women specifically. Since EAR is slightly higher for pregnant women aged 31-50 years (i.e. $300 \mathrm{mg}$ ), calculated percentages below EAR represent a conservative estimate.

indicate several nutrient inadequacies for pregnant women in all BMI categories. Higher estimated prevalences of nutrient inadequacy were found for $\mathrm{Fe}$ and vitamin $\mathrm{D}(>90 \%)$, folate (67\%) and vitamin $\mathrm{B}_{6}(25 \%)$, and to a lesser extent for $\mathrm{Mg}$ (16\%), Zn (17\%), Ca (11\%) and vitamin A (10\%). In addition, $36 \%$ of the women in all BMI categories had total fat intakes above the AMDR. Among obese women, we found a significantly higher estimated prevalence of carbohydrate intakes (relative to EI) below the AMDR compared with normal-weight and overweight women (19v. 9\%). Na intakes exceeded the UL for the majority of pregnant women; for those who were obese, the proportion was significantly higher compared with those in other BMI categories (90 v. 77-78\%). In all four BMI categories, median intakes of $\mathrm{K}$ and fibre were below recommended values (Adequate Intake of $4700 \mathrm{mg}$ and $28 \mathrm{~g}$, respectively), whereas median intakes of essential fatty acids (linoleic and $\alpha$-linolenic) were in-line with Adequate Intakes (13 and $1.4 \mathrm{~g}$, respectively). Although there is no specific DRI for cholesterol, the general recommendation is for adults to limit consumption to $300 \mathrm{mg} / \mathrm{d}^{(28)}$. A higher proportion of obese pregnant women had cholesterol intakes above this recommended value compared with normal-weight women ( $51 v .39 \%$; data not shown).

Table 4 presents Pearson's correlations between BMI and nutritional intakes. We detected a positive linear relationship ( $r$ 0.08) between BMI and total intake of energy. Intakes of several nutrients (adjusted for energy) were also found to be weakly correlated with BMI. Positive correlations ( $r$ 0.07 to
0.09) with BMI were noted for intakes of total fat, saturated fat, $\mathrm{Na}$ and $\mathrm{Zn}$, whereas negative correlations $(r-0.07$ to -0.15$)$ were observed for carbohydrates, total sugars, dietary fibre, vitamin $\mathrm{B}_{6}$, vitamin $\mathrm{C}, \mathrm{Mg}$ and $\mathrm{K}$. Although there was no significant correlation between BMI and folate ( $\mu$ g dietary folate equivalent), we did notice weak correlations for both folate sources. Folic acid, the synthetic form found in fortified food, was positively correlated $(r 0 \cdot 10)$ with BMI, whereas naturally occurring folate was negatively correlated $(r-0.07)$ with BMI.

Table 5 presents Pearson's correlations between GWG and nutritional intakes. Energy intake was positively correlated $(r 0.13)$ with total GWG. Total intakes (in absolute values) of nutrients providing energy (proteins, carbohydrates, total fats and various types of fats), cholesterol and several micronutrients (riboflavin, niacin, vitamin $\mathrm{B}_{6}, \mathrm{Ca}, \mathrm{Fe}, \mathrm{P}, \mathrm{Na}$ and $\mathrm{Zn}$ ) also tended to show a positive linear relationship with GWG (see online Supplementary Table S2). When nutrient intakes were adjusted for energy, certain correlations were detected with total GWG; for the most part, these were specific to certain categories of prepregnancy BMI. For underweight women, negative correlations $(r-0.29$ to -0.33$)$ were found between GWG and intakes of dietary fibre, vitamin $\mathrm{C}$, vitamin $\mathrm{B}_{6}$ and $\mathrm{Mg}$. Among normal-weight women, weaker negative correlations $(r-0 \cdot 10)$ were detected between GWG and dietary intakes of folate and vitamin C. Conversely, among overweight women, we detected positive correlations $(r 0 \cdot 17-0 \cdot 18)$ between GWG and dietary intakes of $\mathrm{Fe}$ and $\mathrm{Zn}$. Among obese pregnant 
Table 4. Correlation between prepregnancy BMI and nutritional intakes (adjusted for energy) during pregnancy†

(Pearson's correlation coefficients and $P$ values)

\begin{tabular}{|c|c|c|}
\hline \multirow[b]{2}{*}{ Nutritional intakes } & \multicolumn{2}{|c|}{ All $(n 861)$} \\
\hline & $r$ & $P$ \\
\hline Energy (kJ (kcal)) & 0.08 & $0.02^{*}$ \\
\hline Protein (\%E) & 0.06 & 0.10 \\
\hline Carbohydrate (\%E) & -0.11 & $0.00^{*}$ \\
\hline Total sugar (\%E) & -0.07 & $0.04^{*}$ \\
\hline Dietary fibre (g/4184 kJ (g/1000 kcal)) & -0.15 & $0.00^{*}$ \\
\hline Total fat $(\% \mathrm{E})$ & 0.07 & $0.04^{*}$ \\
\hline SFA (\%E) & 0.08 & $0.02^{*}$ \\
\hline MUFA (\%E) & 0.02 & 0.47 \\
\hline PUFA (\%E) & 0.03 & 0.35 \\
\hline Cholesterol (mg/4184 kJ (mg/1000 kcal)) & 0.02 & 0.53 \\
\hline Vitamin A (RAE $(\mu \mathrm{g} / 4184 \mathrm{~kJ}(\mu \mathrm{g} / 1000 \mathrm{kcal}))$ & 0.01 & 0.88 \\
\hline Thiamine $(\mathrm{mg} / 4184 \mathrm{~kJ}(\mathrm{mg} / 1000 \mathrm{kcal}))$ & -0.03 & 0.41 \\
\hline Riboflavin (mg/4184 kJ (mg/1000 kcal)) & 0.05 & 0.18 \\
\hline Niacin (NE/4184 kJ (NE/1000 kcal)) & 0.02 & 0.59 \\
\hline Vitamin $B_{6}(\mathrm{mg} / 4184 \mathrm{~kJ}(\mathrm{mg} / 1000 \mathrm{kcal}))$ & -0.08 & $0.02^{*}$ \\
\hline Folate (DFE, $\mu \mathrm{g} / 4184 \mathrm{~kJ}(\mu \mathrm{g} / 1000 \mathrm{kcal}))$ & 0.04 & 0.22 \\
\hline Folic acid $(\mu \mathrm{g})$ & 0.10 & $0.00^{*}$ \\
\hline Folate - natural $(\mu \mathrm{g})$ & -0.07 & $0.04^{*}$ \\
\hline Vitamin $B_{12}(\mu \mathrm{g} / 4184 \mathrm{~kJ}(\mu \mathrm{g} / 1000 \mathrm{kcal}))$ & 0.00 & 0.92 \\
\hline Vitamin C (mg/4184 kJ (mg/1000 kcal)) & -0.12 & $0.00^{*}$ \\
\hline Vitamin D $(\mu \mathrm{g} / 4184 \mathrm{~kJ}(\mu \mathrm{g} / 1000 \mathrm{kcal}))$ & 0.00 & 0.89 \\
\hline $\mathrm{Ca}(\mathrm{mg} / 4184 \mathrm{~kJ}(\mathrm{mg} / 1000 \mathrm{kcal}))$ & 0.03 & 0.38 \\
\hline $\mathrm{Fe}(\mathrm{mg} / 4184 \mathrm{~kJ}(\mathrm{mg} / 1000 \mathrm{kcal}))$ & -0.05 & 0.15 \\
\hline $\mathrm{Mg}(\mathrm{mg} / 4184 \mathrm{~kJ}(\mathrm{mg} / 1000 \mathrm{kcal}))$ & -0.13 & $0.00^{*}$ \\
\hline Phosphorus (mg/4184 kJ (mg/1000 kcal)) & 0.01 & 0.73 \\
\hline $\mathrm{Na}(\mathrm{mg} / 4184 \mathrm{~kJ}(\mathrm{mg} / 1000 \mathrm{kcal}))$ & 0.09 & $0.01^{*}$ \\
\hline $\mathrm{K}(\mathrm{mg} / 4184 \mathrm{~kJ}(\mathrm{mg} / 1000 \mathrm{kcal}))$ & $-0 \cdot 10$ & $0.00^{*}$ \\
\hline $\mathrm{Zn}(\mathrm{mg} / 4184 \mathrm{~kJ}(\mathrm{mg} / 1000 \mathrm{kcal}))$ & 0.08 & $0.02^{*}$ \\
\hline
\end{tabular}

$\mathrm{E}$, energy; RAE, retinol activity equivalent; NE, niacin equivalent; DFE, dietary folate equivalent.

* $P<0.05$.

$\dagger$ Acceptable reporters only (ratio of energy intake:BMR between 1.00 and 2.40 ).

women, positive correlations $(r 0.24)$ with GWG were found for intakes of total fat and saturated fat.

\section{Discussion}

\section{Diet and prepregnancy $B M I$}

One objective of this study was to compare the adequacy of nutritional intakes during pregnancy among women with a different prepregnancy weight status. Our study identified several nutrient inadequacies. Although this observation is generalisable across all prepregnancy BMI categories, we found that a higher proportion of obese women had Na intakes above the UL, carbohydrate intakes (relative to EI) below the AMDR and higher-than-recommended cholesterol intakes compared with women in lower BMI categories.

Previous research on participants of the 3D Cohort Study showed that the vast majority of pregnant women used a multivitamin supplement during pregnancy, which reduced the prevalence of inadequate intake to below $10 \%$ for most micronutrients ${ }^{(16)}$. As there was no significant difference in supplement use during pregnancy across prepregnancy BMI categories (data not shown), we may reasonably expect supplement use to contribute similarly to total nutritional intakes across all prepregnancy BMI categories.
By contrast, we found median intakes of fibre and $\mathrm{K}$ lower than reference values, total fat intake above the AMDR for a certain proportion of pregnant women and $\mathrm{Na}$ intakes above the UL for a majority of women. These intake levels, thus, remain a source of concern. Although these observations were noted in all prepregnancy BMI categories, obese pregnant women were more likely than other pregnant women to have $\mathrm{Na}$ intakes in excess of the UL. Moreover, prevalence estimates of carbohydrates intakes below the AMDR were lower than $10 \%$ in all BMI categories, except for obese women. Our observations for cholesterol intakes among obese women suggest that lower carbohydrate intakes are compensated for by higher fat and protein intakes from animal-food sources.

Future in-depth research on the dietary patterns of 3D Cohort Study participants will allow deriving more specific information on food intake during pregnancy. Still, our findings reinforce the importance of dietary guidance for pregnant women who enter pregnancy with a BMI $\geq 30 \mathrm{~kg} / \mathrm{m}^{2}$ as a means for ensuring appropriate macronutrient intake distribution in overall dietary intake and a higher fibre intake throughout pregnancy. High $\mathrm{Na}$ intakes combined with low $\mathrm{K}$ intakes, which are associated with higher hypertension risks, have been observed for the entire Canadian population ${ }^{(29)}$. These intake levels suggest that nutritional counselling, including strategies to help achieve a better $\mathrm{Na}-\mathrm{K}$ balance in dietary intakes, should also be part of prenatal interventions for all pregnant women, and to an even greater extent for obese women.

We did not find a comparable study reporting adequacy (relative to DRI) of nutritional intakes during pregnancy for prepregnancy BMI categories. A US study conducted between 1995 and 2000 ( $n$ 2394) offered only partially comparable information. It used a self-administered FFQ to derive an index of diet quality (Diet Quality Index for Pregnancy (DQI-P)) ${ }^{(19)}$. Analysis of specific components of the DQI-P indicated that as prepregnancy BMI status increased, the proportion of pregnant women having intakes below the EAR for Fe and folate also generally increased ${ }^{(19)}$. Our analyses did not show significant differences across prepregnancy BMI categories for adequacy of $\mathrm{Fe}$ and folate intakes. Differences in dietary assessment methods, in the timing of the studies, and in study participant characteristics could have contributed to these divergent observations, although both studies appear in agreement on the generally low compliance with nutritional recommendations (DRI) for Fe and folate during pregnancy. Another study conducted in Ireland on obese pregnant women ( $n$ 100; $n 75$ in analyses excluding under-reporters) reported that a substantial proportion had low intakes of carbohydrates and high intakes of fats, particularly saturated fats (as \%E); inadequate dietary intakes of certain key micronutrients (e.g. folate, $\mathrm{Fe}, \mathrm{Ca}$, vitamin D); and high consumption of energy-dense foods rich in fat and sugar ${ }^{(30)}$. Our findings appear to be consistent with these observations and support the notion that women having excess weight before pregnancy may be particularly vulnerable nutritionally.

This conclusion appears to be further supported by the results of our analysis of correlations between prepregnancy BMI and energy-adjusted nutrient intakes during pregnancy. Energy intake was found to be weakly but positively associated 


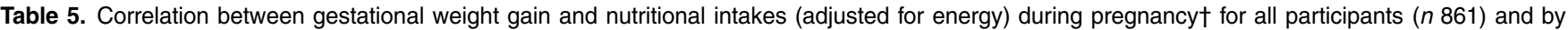
prepregnancy BMI categorył

(Pearson's correlation coefficients and $P$ values)

\begin{tabular}{|c|c|c|c|c|c|c|c|c|c|c|}
\hline \multirow[b]{3}{*}{ Nutritional intakes } & & & \multicolumn{8}{|c|}{ Prepregnancy BMI category } \\
\hline & \multicolumn{2}{|c|}{ All } & \multicolumn{2}{|c|}{ Underweight ( $n$ 51) } & \multicolumn{2}{|c|}{ Normal weight $(n 575)$} & \multicolumn{2}{|c|}{ Overweight ( $n$ 147) } & \multicolumn{2}{|c|}{ Obese $(n 88)$} \\
\hline & $r$ & $P$ & $r$ & $P$ & $r$ & $P$ & $r$ & $P$ & $r$ & $P$ \\
\hline Energy (kJ (kcal)) & 0.13 & $0.00^{*}$ & $0 \cdot 16$ & 0.26 & $0 \cdot 10$ & $0.02^{*}$ & 0.25 & $0.00^{*}$ & 0.22 & $0.04^{*}$ \\
\hline Protein $(\% \mathrm{E})$ & -0.02 & 0.56 & 0.00 & 0.98 & 0.02 & 0.56 & 0.01 & 0.91 & -0.18 & 0.10 \\
\hline Carbohydrate (\%E) & -0.04 & 0.30 & -0.15 & 0.29 & -0.05 & 0.24 & -0.04 & 0.61 & -0.11 & 0.30 \\
\hline Total sugar (\%E) & -0.01 & 0.67 & -0.16 & 0.27 & -0.02 & 0.66 & -0.03 & 0.74 & -0.06 & 0.60 \\
\hline Dietary fibre $(\mathrm{g} / 4184 \mathrm{~kJ}(\mathrm{~g} / 1000 \mathrm{kcal}))$ & -0.02 & 0.51 & -0.30 & $0.03^{*}$ & -0.06 & 0.17 & 0.02 & 0.81 & 0.01 & 0.92 \\
\hline Total fat (\%E) & $0 . .05$ & 0.13 & 0.11 & 0.44 & 0.04 & 0.37 & 0.04 & 0.62 & 0.24 & $0.02^{*}$ \\
\hline SFA (\%E) & 0.05 & 0.14 & 0.16 & 0.27 & 0.05 & 0.20 & -0.01 & 0.89 & 0.24 & $0.03^{*}$ \\
\hline MUFA (\%E) & 0.03 & 0.40 & $0 \cdot 10$ & 0.50 & -0.01 & 0.89 & 0.07 & 0.43 & $0 \cdot 17$ & 0.11 \\
\hline PUFA (\%E) & 0.05 & 0.17 & -0.11 & 0.44 & 0.06 & 0.18 & 0.05 & 0.53 & $0 \cdot 10$ & 0.34 \\
\hline Cholesterol (mg/4184 kJ (mg/1000 kcal)) & 0.03 & 0.43 & 0.10 & 0.50 & 0.03 & 0.44 & 0.03 & 0.76 & 0.02 & 0.85 \\
\hline Vitamin A (RAE, $\mu \mathrm{g} / 4184 \mathrm{~kJ}(\mu \mathrm{g} / 1000 \mathrm{kcal}))$ & 0.01 & 0.70 & 0.08 & 0.56 & -0.03 & 0.48 & 0.07 & 0.41 & 0.00 & 0.97 \\
\hline Thiamine $(\mathrm{mg} / 4184 \mathrm{~kJ}(\mathrm{mg} / 1000 \mathrm{kcal}))$ & -0.03 & 0.41 & -0.12 & 0.40 & -0.07 & 0.09 & 0.05 & 0.56 & 0.12 & 0.26 \\
\hline Riboflavin (mg/4184 kJ (mg/1000 kcal)) & 0.00 & 0.96 & 0.19 & $0 \cdot 17$ & -0.02 & 0.60 & 0.04 & 0.60 & 0.00 & 0.98 \\
\hline Niacin (NE/4184 kJ (NE/1000 kcal)) & 0.00 & 0.94 & -0.12 & 0.41 & 0.02 & 0.63 & 0.08 & 0.35 & -0.08 & 0.45 \\
\hline Vitamin $B_{6}(\mathrm{mg} / 4184 \mathrm{~kJ}(\mathrm{mg} / 1000 \mathrm{kcal}))$ & -0.02 & 0.51 & -0.29 & $0.04^{*}$ & -0.02 & 0.61 & 0.00 & 0.97 & -0.02 & 0.88 \\
\hline Folate (DFE, $\mu \mathrm{g} / 4184 \mathrm{~kJ}(\mu \mathrm{g} / 1000 \mathrm{kcal}))$ & -0.06 & 0.08 & 0.03 & 0.85 & $-0 \cdot 10$ & $0.02^{*}$ & 0.08 & 0.35 & -0.05 & 0.68 \\
\hline Vitamin $B_{12}(\mu \mathrm{g} / 4184 \mathrm{~kJ}(\mu \mathrm{g} / 1000 \mathrm{kcal}))$ & 0.01 & 0.68 & 0.11 & 0.42 & -0.03 & 0.47 & 0.10 & 0.22 & -0.13 & 0.25 \\
\hline Vitamin C (mg/4184 kJ (mg/1000 kcal)) & -0.08 & $0.01^{*}$ & -0.33 & $0.02^{*}$ & $-0 \cdot 10$ & $0.01^{*}$ & $-0 \cdot 13$ & 0.12 & 0.07 & 0.54 \\
\hline Vitamin D $(\mu \mathrm{g} / 4184 \mathrm{~kJ}(\mu \mathrm{g} / 1000 \mathrm{kcal}))$ & -0.02 & 0.59 & -0.01 & 0.92 & -0.02 & 0.72 & -0.07 & 0.42 & 0.05 & 0.66 \\
\hline $\mathrm{Ca}(\mathrm{mg} / 4184 \mathrm{~kJ}(\mathrm{mg} / 1000 \mathrm{kcal}))$ & 0.01 & 0.71 & 0.15 & 0.30 & 0.02 & 0.61 & -0.02 & 0.85 & -0.02 & 0.89 \\
\hline $\mathrm{Fe}(\mathrm{mg} / 4184 \mathrm{~kJ}(\mathrm{mg} / 1000 \mathrm{kcal}))$ & 0.00 & 0.94 & -0.10 & 0.47 & -0.05 & 0.23 & 0.17 & $0.04^{*}$ & 0.03 & 0.75 \\
\hline $\mathrm{Mg}(\mathrm{mg} / 4184 \mathrm{~kJ}(\mathrm{mg} / 1000 \mathrm{kcal}))$ & -0.04 & 0.20 & -0.29 & $0.04^{*}$ & -0.08 & 0.05 & 0.04 & 0.66 & -0.03 & 0.81 \\
\hline $\mathrm{P}(\mathrm{mg} / 4184 \mathrm{~kJ}(\mathrm{mg} / 1000 \mathrm{kcal}))$ & -0.01 & 0.76 & 0.01 & 0.93 & 0.00 & 0.99 & 0.02 & 0.84 & -0.09 & 0.39 \\
\hline $\mathrm{Na}(\mathrm{mg} / 4184 \mathrm{~kJ}(\mathrm{mg} / 1000 \mathrm{kcal}))$ & 0.04 & 0.25 & 0.05 & 0.73 & 0.08 & 0.06 & 0.10 & 0.24 & -0.03 & 0.78 \\
\hline $\mathrm{K}(\mathrm{mg} / 4184 \mathrm{~kJ}(\mathrm{mg} / 1000 \mathrm{kcal}))$ & -0.05 & 0.14 & -0.24 & 0.08 & -0.07 & 0.11 & -0.04 & 0.59 & -0.03 & 0.78 \\
\hline $\mathrm{Zn}(\mathrm{mg} / 4184 \mathrm{~kJ}(\mathrm{mg} / 1000 \mathrm{kcal}))$ & 0.04 & 0.22 & 0.27 & 0.06 & 0.06 & $0 \cdot 16$ & 0.18 & $0.03^{*}$ & -0.18 & 0.09 \\
\hline
\end{tabular}

$\mathrm{E}$, energy; RAE, retinol activity equivalent; $\mathrm{NE}$, niacin equivalent; DFE, dietary folate equivalent.

${ }^{*} P<0.05$.

$\dagger$ Acceptable reporters only (ratio of energy intake:BMR between 1.00 and 2.40 ).

‡ Underweight: $\mathrm{BMI}<18.5 \mathrm{~kg} / \mathrm{m}^{2}$; normal weight: $18.5 \mathrm{~kg} / \mathrm{m}^{2} \leq \mathrm{BMl}<25.0 \mathrm{~kg} / \mathrm{m}^{2}$; overweight: $25.0 \mathrm{~kg} / \mathrm{m}^{2} \leq \mathrm{BMl}<30.0 \mathrm{~kg} / \mathrm{m}^{2} ;$ obese: $\mathrm{BMl} \geq 30 \cdot 0 \mathrm{~kg} / \mathrm{m}^{2}$.

with prepregnancy BMI. Analyses of energy-adjusted nutrient intakes by prepregnancy BMI suggested that women with different weight statuses before pregnancy also differed in dietary intake profiles while pregnant. A higher BMI before pregnancy was associated with higher intakes of certain nutrients found in animal-based sources (e.g. total fat, saturated fat, $\mathrm{Zn}$ ) or processed (e.g. Na) and enriched (e.g. folic acid) food products. By comparison, a lower BMI before pregnancy was associated with higher intakes of carbohydrates (including total sugars), dietary fibre and nutrients such as naturally occurring folate, vitamin $\mathrm{C}$, vitamin $\mathrm{B}_{6}, \mathrm{~K}$ and $\mathrm{Mg}$. This combination of nutrients suggests a higher consumption of plant-based food sources, such as vegetables, fruit, legumes and whole-grain products among women with lower BMI

Derbyshire et al. ${ }^{(23)}$ have looked at correlations between prepregnancy BMI and nutritional intakes (in absolute values) in the first trimester of pregnancy. Their study was conducted on a small sample of pregnant women in London, UK ( $n 72$; $n 51$ in analyses excluding under-reporters). Once underreporters were removed from the analyses, the study detected no association between BMI and energy or nutrient intakes, except for folate (i.e. prepregnancy BMI was negatively correlated with folate intake). Another, larger study ( $n$ 795), which used nutritional data collected among pregnant women who participated in the US National Health and Nutrition
Examination Survey between 2003 and 2012, did not find associations between prepregnancy weight status and intakes of energy or major macronutrients as $\% \mathrm{E}^{(17)}$. This study used single-day dietary recall to derive an overall diet quality score (Healthy Eating Index (HEI)-2010). It did not consider the misreporting of energy intakes in reported results. The authors did note, however, that obese women before pregnancy tended to have a lower score for the Na component of the HEI-2010 compared with normal-weight women ${ }^{(17)}$. This result is indicative of higher intakes of $\mathrm{Na} / 4184 \mathrm{~kJ}$ (1000 kcal) for obese women. Conversely, dietary intakes of folate and $\mathrm{Fe} / 4184 \mathrm{~kJ}$ $(1000 \mathrm{kcal})$ were found to be significantly lower for obese women than for underweight women ${ }^{(17)}$. The results for $\mathrm{Na}$ appear to accord with our observations. Our study detected associations between folate intake (on a per $4184 \mathrm{~kJ}$ (1000 kcal) basis) and prepregnancy BMI only for specific sources of folate (i.e. an inverse association was found for folate naturally occurring in food and a positive association found for folic acid, which is present only in fortified food).

\section{Diet and gestational weight gain}

Our findings show that adherence to GWG recommendations posed problems for many pregnant women. One in six did not gain enough weight during pregnancy according to IOM 
recommendations, whereas almost one in two exceeded the BMI-specific GWG recommendation. The purpose of our study was to explore potential associations between nutritional intakes during pregnancy and GWG. Overall, our analyses showed a positive correlation between total intakes of energy and nutrients, and GWG. The relationship between energy intake and GWG is consistent with the conclusions of two recent systematic reviews of observational studies in industrialised countries ${ }^{(31,32)}$. Because intakes of many nutrients are correlated with energy intakes ${ }^{(33)}$, pregnant women with highenergy intakes are also more likely to ingest higher absolute quantities of nutrients, which may explain a similar positive association with GWG for several nutrients in absolute values. We did not, however, detect any association between macronutrient composition (as \%E) and GWG, except for total fat and saturated fat specific to obese pregnant women.

Other studies examining dietary macronutrient composition during pregnancy relative to GWG have yielded inconclusive results $^{(32)}$. Recent systematic reviews, moreover, have shown considerable variability in the attributes of studies on macronutrient composition relative to GWG (e.g. in the measurement and definition of GWG; in dietary assessment methods; in the types of analyses) ${ }^{(31,32)}$. This methodological inconsistency might explain irregular associations between GWG and intakes of protein, carbohydrates, and total fat, independent of energy content. Nevertheless, associations may well exist for certain types of fat (e.g. saturated fat ${ }^{(32)}$ ) or may be specific to certain subgroups (e.g. overweight or obese women) ${ }^{(32)}$, all of which would accord with our findings.

When considering energy-adjusted (i.e. per $4184 \mathrm{~kJ}$ $(1000 \mathrm{kcal}))$ intakes of other nutrients, we observed specific patterns of association between GWG and certain prepregnancy BMI categories. For women in lower BMI categories $\left(<25 \mathrm{~kg} / \mathrm{m}^{2}\right)$, and particularly for underweight women $\left(\mathrm{BMI}<18 \mathrm{~kg} / \mathrm{m}^{2}\right)$, lower GWG was associated with higher energy-adjusted intakes for certain nutrients commonly found in plant-based food sources such as fibre, vitamin C, vitamin $\mathrm{B}_{6}$, $\mathrm{Mg}$ or folate. These results may suggest a propensity toward health-conscious dietary choices among underweight or normal-weight women who have low GWG. By contrast, for overweight women $\left(25 \mathrm{~kg} / \mathrm{m}^{2} \leq \mathrm{BMI}<30 \mathrm{~kg} / \mathrm{m}^{2}\right)$, higher GWG was associated with higher intakes (energy adjusted) of both $\mathrm{Zn}$ and $\mathrm{Fe}$, which could result from a propensity toward higher consumption of animal-based sources of protein (e.g. meat) among overweight women who have high GWG. More extensive research into the dietary patterns of our study participants will allow examining food intake during pregnancy relative to GWG in greater detail. In any case, our analyses of nutritional intakes to date already indicate the existence of differential relationships between diet and GWG across prepregnancy BMI categories, as other studies have likewise suggested $^{(32,34)}$.

\section{Strengths and limitations}

Participants in the 3D Cohort Study were recruited from a variety of geographical areas in the Canadian province of Quebec. Our sample was not, however, representative of the
Quebec population. Almost two-thirds of participants had university degrees and half came from high-income families, which indicates a higher socio-economic status than that of the general population. Our study findings may also not be generalisable to preterm pregnancies as we included only participants who delivered at 37 weeks or later. Because participants were recruited in the first trimester of pregnancy, reported prepregnancy weights were used to determine prepregnancy weight status and to assess total GWG. Reported anthropometric data are frequently used in pregnancy/birth cohorts because recruiting women for pregnancy-related studies before they become pregnant can prove challenging in countries that have low birth rates. Despite later-than-ideal recruitments, a recent study conducted in the USA concluded that prepregnancy weight statuses estimated from self-reported data are reliable and valid for population-based studies ${ }^{(35)}$.

We collected detailed dietary data from pregnant women using a 3-d food record. In a recent systematic review of the validity of methods for assessing dietary intakes during pregnancy, food records proved to have the highest validity vis-à-vis biomarkers ${ }^{(36)}$. Although most dietary assessment methods that rely on self-reporting are prone to energy under-reporting ${ }^{(37)}$, we minimised the impact of misreported dietary information by estimating misreporting levels in our sample and by analysing only data provided by plausible reporters.

Our study presents an in-depth assessment of dietary intake in the second trimester of pregnancy for a large number of women. During the second trimester, pregnant women are less likely to experience the physical distress that often characterises early pregnancy (e.g. vomiting) and late pregnancy (e.g. heartburn). In the third trimester, certain complications (preeclampsia, diabetes) can alter participants' diets, and premature births can prompt women to withdraw from studies. Despite its comprehensive scope and extensive database, our portrait of dietary intake may not be representative of participant's diets throughout an entire pregnancy. One study of overweight and obese pregnant women has indicated that dietary quality tends to decrease throughout pregnancy ${ }^{(38)}$. Other studies, however, have reported that diet composition and dietary patterns, whether before and during pregnancy or between trimesters, vary little $\mathrm{e}^{(39-41)}$. To gain a fuller understanding of dietary changes throughout pregnancy, more prospective dietary studies (which ideally would include the preconceptional period) are warranted.

\section{Conclusion}

Our assessment of nutritional intakes among pregnant women revealed several nutrient inadequacies generalisable across all prepregnancy BMI categories. We also observed that higher proportions of obese women had carbohydrate intakes (adjusted for energy) below the acceptable range and $\mathrm{Na}$ intakes above the UL. Our analyses suggest that differential relationships between diet and GWG exist across all prepregnancy BMI categories. Overall, our findings indicate that nutrition interventions are needed to help pregnant women achieve their optimal GWG while also meeting their nutritional requirements. 


\section{Acknowledgements}

The authors are grateful to the 3D participants who took part in the nutrition study during their pregnancy and to members of the 3D study who contributed to data collection and coding. The authors also thank Robert Sullivan for assisting with manuscript editing.

This work was supported by the Canadian Institutes of Health Research (CIHR) (L. D., grant no. MOP 133422), (W. D. F., grant no. CRI 88413). CIHR had no role in the design, analysis or writing of this article.

L. D. designed the nutrition study, directed the analyses and interpretation of data, and had primary responsibility for final content. M. D. was responsible for all statistical analyses. L. D. and B. F.-B. wrote the paper. C. K. C., B. F.-B., R. E. T. contributed to study design and results interpretation. W. D. F. is the director of the 3D Cohort Study and was incharge of data collection and the overall direction of the study. All authors made critical revisions to the text and approved the manuscript submitted for publication.

None of the authors has any conflicts of interest to declare.

\section{Supplementary material}

For supplementary material/s referred to in this article, please visit https://doi.org/10.1017/S0007114518001393

\section{References}

1. Organisation for Economic Co-operation and Development Directorate for Employment, Labour and Social Affairs (2014) Obesity update. June. http://www.oecd.org/health/ObesityUpdate-2014.pdf (accessed November 2016).

2. Statistics Canada (2014) Body composition of adults, 2012 to 2013. Health Fact Sheets 82-625-X. http://www.statcan. gc.ca/pub/82-625-x/2014001/article/14104-eng.htm (accessed November 2016).

3. Leddy MA, Power ML \& Schulkin J (2008) The impact of maternal obesity on maternal and fetal health. Rev Obstet Gynecol 1, 170-178.

4. Nutrition Working Group, O'Connor DL, Blake J, et al. (2016) Canadian consensus on female nutrition: adolescence, reproduction, menopause and beyond. J Obstet Gynaecol Can 38, 508-554.

5. Institute of Medicine (US) \& National Research Council (US) Committee to Reexamine Institute of Medicine Pregnancy Weight Guidelines, Rasmussen KM \& Yaktine AL editors (2009) Weight Gain During Pregnancy: Reexamining the Guidelines. Washington, DC: National Academies Press.

6. Ferraro ZM, Barrowman N, Prud'homme D, et al. (2012) Excessive gestational weight gain predicts large for gestational age neonates independent of maternal body mass index. J Matern Fetal Neonatal Med 25, 538-542.

7. Deputy NP, Sharma AJ \& Kim SY (2015) Gestational weight gain - United States, 2012 and 2013. MMWR Morb Mortal Wkly Rep 64, 1215-1220.

8. Dzakpasu S, Fahey J, Kirby RS, et al. (2015) Contribution of prepregnancy body mass index and gestational weight gain to adverse neonatal outcomes: population attributable fractions for Canada. BMC Pregnancy Childbirth 15, 21.

9. Guelinckx I, Devlieger R, Beckers K, et al. (2008) Maternal obesity: pregnancy complications, gestational weight gain and nutrition. Obes Rev 9, 140-150.
10. Godfrey KM, Gluckman PD \& Hanson MA (2010) Developmental origins of metabolic diseases: lifecourse and intergenerational perspectives. Trends Endocrinol Metab 21, 199-205.

11. Yajnik CS \& Deshmakh US (2008) Maternal nutrition, intrauterine programming and consequential risks in the offspring. Rev Endocr Metab Disord 9, 203-211.

12. Health Canada (2010) Prenatal nutrition guidelines for health professionals. Gestational weight gain. http://www.hcsc.gc.ca/fn-an/alt_formats/pdf/nutrition/prenatal/ewba-mbsaeng.pdf (accessed November 2016).

13. Institute of Medicine (2006) Dietary Reference Intakes: The Essential Guide to Nutrient Requirements. Washington, DC: National Academies Press.

14. Blumfield ML, Hure AJ, Macdonald-Wicks L, et al. (2012) Systematic review and meta-analysis of energy and macronutrient intakes during pregnancy in developed countries. Nutr Rev 70, 322-336.

15. Blumfield ML, Hure AJ, Macdonald-Wicks L, et al. (2013) A systematic review and meta-analysis of micronutrient intakes during pregnancy in developed countries. Nutr Rev 71, 118-132.

16. Dubois L, Diasparra M, Bédard B, et al. (2017) Adequacy of nutritional intake from food and supplements in a cohort of pregnant women in Québec, Canada: the 3D Cohort Study (Design, Develop, Discover). Am J Clin Nutr 10, 541-548.

17. Shin D, Lee KW \& Song WO (2016) Pre-pregnancy weight status is associated with diet quality and nutritional biomarkers during pregnancy. Nutrients $\mathbf{8}, 162$.

18. McGowan CA \& McAuliffe FM (2013) Maternal dietary patterns and associated nutrient intakes during each trimester of pregnancy. Public Health Nutr 16, 97-107.

19. Laraia BA, Bodnar LM \& Siega-Riz AM (2007) Pregravid body mass index is negatively associated with diet quality during pregnancy. Public Health Nutr 10, 920-926.

20. Rifas-Shiman SL, Rich-Edwards JW, Kleinman KP, et al. (2009) Dietary quality during pregnancy varies by maternal characteristics in Project Viva: a US cohort. J Am Diet Assoc 109, 1004-1011.

21. Tsigga M, Filis V, Hatzopoulou K, et al. (2011) Healthy Eating Index during pregnancy according to pre-gravid and gravid weight status. Public Health Nutr 14, 290-296.

22. Shin D, Bianchi L, Chung H, et al. (2014) Is gestational weight gain associated with diet quality during pregnancy? Matern Child Health J 18, 1433-1443.

23. Derbyshire E, Davies J, Costarelli V, et al. (2006) Prepregnancy body mass index and dietary intake in the first trimester of pregnancy. J Hum Nutr Diet 19, 267-273.

24. Fraser WD, Shapiro GD, Audibert F, et al. (2016) 3D Cohort Study: The Integrated Research Network in Perinatology of Quebec and Eastern Ontario. Paediatr Perinat Epidemiol 30, 623-632.

25. Health Canada (2010) Canadian Nutrient File (version 2010). Ottawa: Nutrition Research Division.

26. Black A (2000) Critical evaluation of energy intake using the Goldberg cut-off for energy intake: basal metabolic rate. A practical guide to its calculation, use and limitations. Int J Obes Relat Metab Disord 24, 1119-1130.

27. Tooze JA, Kipnis V, Buckman DW, et al. (2010) A mixed-effects model approach for estimating the distribution of usual intake of nutrients: the NCI method. Stat Med 29, 2857-2868.

28. World Health Organization and Food and Agriculture Organization of the United Nations (2003) Diet, Nutrition and the Prevention of Chronic Diseases, Report of a Joint WHO/FAO Expert Consultation. WHO Technical Report Series, no. 916. Geneva: WHO.

29. Tanase CM, Koski KG, Laffey PJ, et al. (2011) Canadians continue to consume too much sodium and not enough potassium. Can J Public Health 102, 164-168. 
30. Lindsay KL, Heneghan C, McNulty B, et al. (2015) Lifestyle and dietary habits of an obese pregnant cohort. Matern Child Health J 19, 25-32.

31. Streuling I, Beyerlein A, Rosenfeld E, et al. (2011) Weight gain and dietary intake during pregnancy in industrialized countries - a systematic review of observational studies. I Perinat Med 39, 123-129.

32. Tielemans MJ, Garcia AH, Peralta Santos A, et al. (2016) Macronutrient composition and gestational weight gain: a systematic review. Am J Clin Nutr 103, 83-99.

33. Willett WC, Howe GR \& Kushi LH (1997) Adjustment for total energy intake in epidemiologic studies. Am J Clin Nutr $\mathbf{6 5}$, 1220S-1228S

34. Hillesund ER, Bere E, Haugen M, et al. (2014) Development of a New Nordic Diet score and its association with gestational weight gain and fetal growth - a study performed in the Norwegian Mother and Child Cohort Study (MoBa). Public Health Nutr 17, 1909-1918.

35. Shin D, Chung H, Weatherspoon L, et al. (2014) Validity of prepregnancy self-reported height and weight. Matern Child Health J 18, 1667-1674.
36. Vézina-Im LA \& Robitaille J (2014) Validity and reliability of self-reported measures of foods and nutrients in pregnancy: a systematic review. Curr Nutr Rep 3, 245-280.

37. Subar AF, Freedman LS, Tooze JA, et al. (2015) Addressing current criticism regarding the value of self-report dietary data. J Nutr 145, 2639-2645.

38. Moran LJ, Sui Z, Cramp CS, et al. (2013) A decrease in diet quality occurs during pregnancy in overweight and obese women which is maintained post-partum. Int J Obes (Lond) 37, 704-711.

39. Talai Rad N, Ritterath C, Siegmund T, et al. (2011) Longitudinal analysis of changes in energy intake and macronutrient composition during pregnancy and 6 weeks post-partum. Arch Gynecol Obstet 283, 185-190.

40. Cucó G, Fernández-Ballart J, Sala J, et al. (2006) Dietary patterns and associated lifestyles in preconception, pregnancy and postpartum. Eur J Clin Nutr 60, 364-371.

41. Crozier SR, Robinson SM, Godfrey KM, et al. (2009) Women's dietary patterns change little from before to during pregnancy. J Nutr 139, 1956-1963. 